\title{
NOUVELLe
}

\section{Reprogrammer les cellules de patients parkinsoniens pour révéler de nouvelles marques pathologiques de la maladie}

Emmanuel Nivet ${ }^{1}$, Guang-hui Liu ${ }^{1,2}$, Nuria Montserrat ${ }^{3}$, Juan Carlos Izpisua Belmonte ${ }^{1,3}$

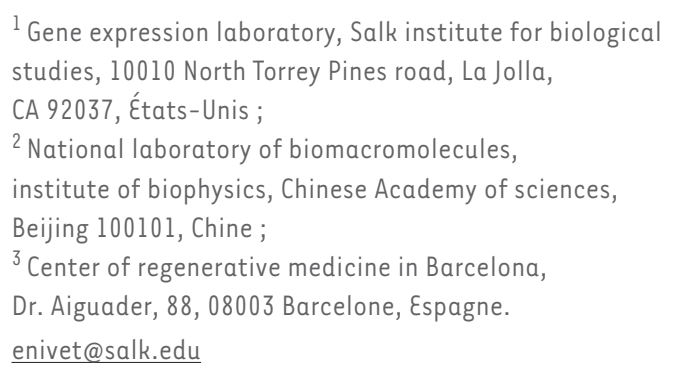
studies, 10010 North Torrey Pines road, La Jolla, CA 92037, États-Unis ;

${ }^{2}$ National laboratory of biomacromolecules, institute of biophysics, Chinese Academy of sciences, Beijing 100101, Chine ;

${ }^{3}$ Center of regenerative medicine in Barcelona, enivet@salk.edu

> La reprogrammation cellulaire (ou comment changer l'identité d'une cellule) est une approche pleine de promesses pour l'étude des maladies et le développement de nouveaux outils thérapeutiques. Historiquement, les travaux fondateurs de Briggs et King [1], puis de Gurdon [2] et enfin de Takahashi et yamanaka [3] ont établi les connaissances aboutissant à la possibilité de reprogrammer une cellule afin qu'elle acquière les caractéristiques d'une cellule souche embryonnaire pluripotente [11]. L'avènement des cellules souches pluripotentes induites humaines (aussi appelées iPS pour induced pluripotent stem cells) [4] a permis d'envisager la reprogrammation de cellules prélevées sur des patients afin de pouvoir modéliser in vitro le développement de maladies $[5,12]$. Des données convergentes montrent que la reprogrammation vers la pluripotence permet une « remise à zéro » des marques épigénétiques [6] acquises au fur et à mesure du temps, sans profondément altérer les marques génétiques qui, elles, sont ancrées dans le génome de la cellule comme une marque irréversible (à moins que le génome ne soit modifié artificiellement). De plus, il a été démontré que, lorsqu'une cellule pluripotente induite est soumise à certains signaux environnementaux visant à mimer les étapes du développement, des processus de différenciation se mettent en place, et différents types cellulaires peuvent être obtenus, lesquels pourront réacquérir les marques de la maladie [7, 8]. Ainsi, en reprogrammant vers un état « naïf » des cellules porteuses de mutation(s) génique(s), il devient désormais possible de reproduire le développement d'une maladie. Ces modèles permettent d'acquérir de nouvelles connaissances qui pourraient s'avérer importantes pour la compréhension de la pathologie, et donc le développement de nouveaux traitements.

La maladie de Parkinson:

la mutation G2019 du gène LRRK2 comme modèle d'étude

Récemment, nous avons mis à profit ce paradigme expérimental afin d'étudier la maladie de Parkinson [9]. Nous nous sommes plus particulièrement intéressés au gène LRRK2 (leucine-rich repeat kinase 2) et à sa mutation substituant une glycine par une sérine en position 2019 (G2019) ; cette mutation est responsable d'une forme génétique de la maladie de Parkinson. Fait intéressant, de récents travaux ont montré que cette mutation entraîne des perturbations au niveau des centres de la neurogenèse chez la souris [10]. En conséquence, les cellules souches neurales nous sont apparues comme un outil de choix pour étudier les conséquences de cette mutation au niveau cellulaire et/ou moléculaire. Dans un premier temps, nous avons reprogrammé des fibroblastes provenant de biopsies de peau effectuées chez des patients atteints de la maladie de Parkinson et porteurs, ou non, de la mutation G2019. Des iPS ont été obtenues et caractérisées, puis différenciées en cellules souches neurales. Nos premières analyses ne nous ont pas permis d'identifier de différences entre les cellules souches neurales porteuses ou non de la mutation. Nous avons alors émis l'hypothèse qu'il était important, pour provoquer l'apparition d'un comportement pathologique de ces cellules mutées, de dupliquer in vitro la composante temporelle associée in vivo au déclenchement des premiers symptômes de la maladie. Pour ce faire, nous avons maintenu les cellules en culture pendant une longue période en les réensemençant dès que la culture était confluente.

\section{Mimer le vieillissement in vitro démasque de nouvelles marques pathologiques}

Les cellules deviennent dès lors « vieillissantes » au fur et à mesure des passages cellulaires. Nous avons ainsi identifié, à partir du $14^{\mathrm{e}}$ passage, des altérations au niveau du noyau dans les cellules souches neurales porteuses de la mutation G2019. Ces anomalies associaient un élargissement du compartiment nucléaire, des défauts de circularité de l'enveloppe nucléaire et une perte locale d'expression des protéines lamine Bl et B2. Ces paramètres s'aggravaient à mesure des passages alors que les cellules souches neurales issues des iPS de patients 

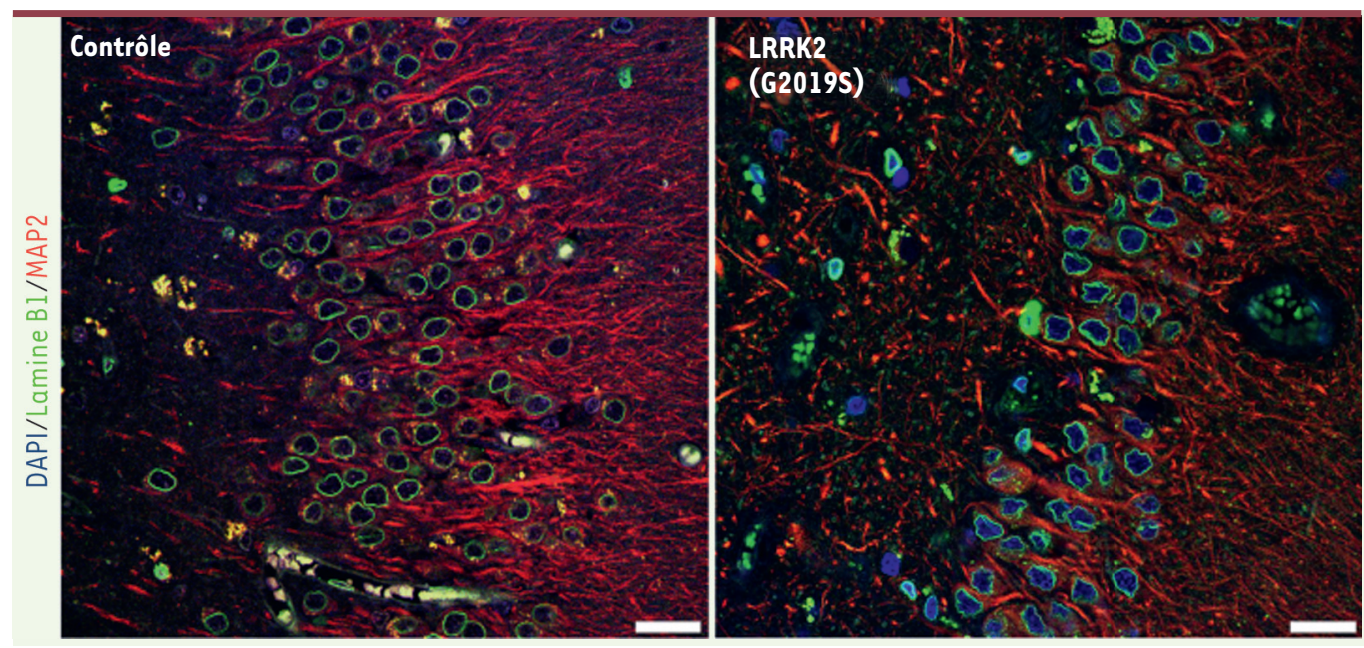

Figure 1. Analyse de tissu cérébral de patients parkinsoniens porteurs de la mutation LRRK2-G2019. Nous avons analysé post-mortem des coupes de cerveaux provenant de patients parkinsoniens porteurs (droite) ou non (gauche) de la mutation G2019 du gène LRRK2. Les analyses immunohistochimiques pour la lamine Bl (une protéine de l'enve-

loppe nucléaire, vert), et la protéine neuronale MAP2 (microtubule-associated protein 2, rouge) révèlent des altérations de la membrane nucléaire au niveau des zones de neurogenèse de l'hippocampe chez les patients parkinsoniens. Les noyaux cellulaires ont été marqués par le DAPI (4',6'-diamidino2-phénylindole, bleu). Barre d'échelle : $25 \mu \mathrm{m}$.

parkinsoniens mais non porteurs de la mutation G2019, étaient parfaitement saines au même stade du repiquage, donc au même «âge ». Nous avons montré que la forme mutée de LRRK2 interagit avec des protéines nucléaires, et notamment les lamines Bl et B2, et que la surexpression de LRRK2-G2019 dans des cellules saines induit des déformations nucléaires. De plus, la forme phosphorylée des protéines lamine B1 et B2 était augmentée dans les cellules porteuses de la mutation, suggérant une activité kinase excessive de LRRK2. Ceci fut en partie confirmé par l'observation, dans les cellules souches neurales mutées qui étaient cultivées pendant de nombreux passages, d'une augmentation de la phosphorylation de la sérine 935 témoignant d'une activation de LRRK2. Associées à l'apparition de ces aberrations nucléaires, d'autres différences entre cellules mutées et non mutées furent observées: (1) des anomalies au niveau de l'hétérochromatine; (2) des modifications des marques épigénétiques ; (3) une capacité réduite de prolifération, et (4) une capacité de différenciation vers le lignage neuronal quasi nulle. Nous avons démontré que nous pouvions corriger la mutation génique dans les iPS-G2019 et ainsi restaurer un phénotype non pathologique. L'inverse est aussi vrai puisque l'induction de la mutation G2019 dans des cellules souches embryonnaires suffit à induire le phénotype aberrant dans les cellules souches neurales différenciées à partir de ces cellules souches embryonnaires et maintenues en culture. Il est très intéressant de noter que l'ajout d'un inhibiteur spécifique de LRRK2 était suffisant pour annuler les effets de la mutation sur les cellules souches neurales.

\section{Plus qu'une observation in vitro, une réalité in vivo!}

Dans le but de confirmer in vivo nos résultats expérimentaux in vitro, nous avons analysé l'architecture nucléaire des cellules neurales sur des échantillons cérébraux prélevés post-mortem sur des patients atteints de la maladie de Parkinson et porteurs de la mutation G2019 (Figure 1). Nous avons observé que de nombreuses cellules présentaient des altérations de leur membrane nucléaire et que celles-ci étaient localisées dans des zones neurogéniques (hippocampe). Ce phénotype aberrant n'était en revanche détecté que dans un très faible nombre de cellules provenant d'échantillons prélevés chez des patients non parkinsoniens au même âge. De façon surprenante, l'analyse d'autres échantillons issus de patients parkinsoniens non porteurs de la mutation révéla, chez certains d'entre eux, un phénotype cellulaire souvent tout aussi anormal que celui observé chez les patients G2019.

En conclusion, les cellules souches pluripotentes induites nous ont permis d'identifier des altérations pathologiques qui n'avaient pas encore été décrites pour la maladie de Parkinson. Ceci montre une fois de plus, s'il en était besoin, la puissance d'un tel outil dans la modélisation des maladies [12]. En effet, c'est la découverte d'un phénotype in vitro qui nous a conduits à identifier son implication dans le processus pathologique in vivo, alors que c'est généralement l'inverse qui se produit. Ainsi, ce type de modélisation in vitro offre une opportunité unique pour améliorer notre compréhension et notre connaissance des mécanismes physiopathologiques. Les plateformes de modélisation ont donc un avenir prometteur. Elles pourraient, en facilitant le criblage de nouvelles molécules thérapeutiques [13], permettre de découvrir de nouvelles stratégies thérapeutiques, en particulier dans les maladies d'origine génétique. $\diamond$ Resetting Parkinson's disease patient-derived cells to unveil new pathological marks

\section{LIENS D'INTÉRÊT}

Les auteurs déclarent n'avoir aucun lien d'intérêt concernant les données publiées dans cet article. 


\section{RÉFÉRENCES}

1. Briggs R, King TJ. Transplantation of living nuclei from blastula cells into enucleated frogs' eggs. Proc Natl Acad Sci USA $1952 ; 38:$ 455-63.

2. Gurdon JB, Elsdale TR, Fischberg M. Sexually mature individuals of Xenopus laevis from the transplantation of single somatic nuclei. Nature 1958 ; $182: 64-5$.

3. Takahashi K, Yamanaka S. Induction of pluripotent stem cells from mouse embryonic and adult fibroblast cultures by defined factors. Cell $2006 ; 126: 663-76$.

4. Takahashi K, Tanabe K, Ohnuki M, et al. Induction of pluripotent stem cells from adult human fibroblasts by defined factors. Cell $2007 ; 131: 861-72$.
5. Tiscornia G, Vivas EL, Izpisua Belmonte JC. Diseases in a dish: modeling human genetic disorders using induced pluripotent cells. Nat Med 2011 ; 17 : 1570-6.

6. Papp B, Plath K. Reprogramming to pluripotency: stepwise resetting of the epigenetic landscape. Cell Res 2011 ; 21 : 486-501.

7. Liu GH, Barkho BZ, Ruiz S, et al. Recapitulation of premature ageing with iPSCs from Hutchinson-Gilford progeria syndrome. Nature $2011 ; 472$ : 221-5.

8. Marchetto MC, Carromeu C, Acab A, et al. A model for neural development and treatment of Rett syndrome using human induced pluripotent stem cells. Cell 2010 ; $143: 527-39$

9. Liu GH, Qu J, Suzuki K, et al. Progressive degeneration of human neural stem cells caused by pathogenic LRRK2. Nature 2012 ; 491 : 603-7.
NOUVELLE

\section{Une avancée technique pour comprendre la dynamique de l'horloge de segmentation}

10. Winner B, Melrose HL, Zhao C, et al. Adult neurogenesis and neurite outgrowth are impaired in LRRK2 G2019S mice. Neurobiol Dis 2011 ; $41: 706-16$.

11. Coulombel L. Reprogrammation nucléaire d'une cellule différenciée : on efface tout et on recommence. Med Sci (Paris) 2007 ; $23: 667-70$.

12. Maury Y, Gauthier M, Peschanski M, Martinat C. Human pluripotent stem cells: opening key for pathological modeling. Med Sci (Paris) 2011 ; 27 : 443-6.

13. Laustriat D, Gide J, Héchard C, Peschanski M. Les cellules souches embryonnaires et le pharmacologue. Med Sci (Paris) 2009 ; 25 (suppl 2) : 32-8.

${ }^{1}$ Laboratoire de biologie moléculaire de la cellule (LBMC), UMR5239, CNRS/UCBL/ENS de Lyon, 46, allée d'Italie, 69007 Lyon, France ;

2 McGill university, Montreal, Québec, Canada ;

${ }^{3}$ University of California at Berkeley, Berkeley, États-Unis ;

${ }^{4}$ The Ohio state university, Columbus, États-Unis.

emilie.delaune-henry@ens-lyon.fr

permettant de visualiser l'expression de gènes cycliques chez la souris. Pour comprendre les mécanismes qui génèrent et synchronisent les oscillations, il est essentiel de visualiser l'activité de l'horloge au niveau cellulaire. Visualiser un processus aussi dynamique grâce à un système rapporteur est délicat car : (1) le rapporteur doit être produit et dégradé dans le court temps d'un cycle de l'horloge (2 h chez la souris, $30 \mathrm{~min}$ chez le poisson zèbre), (2) le rapporteur doit rester détectable malgré sa grande instabilité, et (3) l'activité de l'horloge endogène ne doit pas être affectée par le rapporteur [2]. Les premiers rapporteurs de l'horloge développés chez la souris ont permis de visualiser les vagues d'expression de gènes cycliques au niveau tissulaire [3-5]. Afin d'atteindre un niveau de résolution cellulaire, nous nous sommes tournés vers le modèle du poisson zèbre, dont la transparence et le développement externe sont des atouts pour l'imagerie à haute résolution. Dans un travail publié récemment dans Developmental Cell, nous avons développé un système rapporteur très dynamique de l'horloge, 